\title{
Acute vagal modulation of electrophysiology of the atrial and pulmonary veins increases vulnerability to atrial fibrillation
}

\author{
Mário Oliveira $^{1,2}$, M. Nogueira da Silva ${ }^{3}$, Vera Geraldes ${ }^{1,2}$, Rita Xavier ${ }^{1,2}$, Sérgio Laranjo ${ }^{1,2}$, Vitor Silva ${ }^{1,2}$, \\ Gabriela Postolache ${ }^{1,2}$, Rui Ferreira ${ }^{3}$ and Isabel Rocha ${ }^{1,2}$ \\ ${ }^{1}$ Institute of Physiology, Faculty of Medicine, Lisbon, Portugal \\ ${ }^{2}$ Unit of Autonomic, Nervous System, Instituto de Medicina Molecular, Lisbon, Portugal \\ ${ }^{3}$ Department of Cardiology, Santa Marta Hospital, Lisbon, Portugal
}

\begin{abstract}
Vagal activity is thought to influence atrial electrophysiological properties and play a role in the initiation and maintenance of atrial fibrillation (AF). We evaluated the effects of acute vagal stimulation on atrial conduction, refractoriness of atrial and pulmonary veins (PVs) and inducibility of AF. An open-chest epicardial approach was performed in New Zealand White rabbits with preserved autonomic innervation. Atrial electrograms were obtained with four unipolar electrodes placed epicardially along the atria $(n=22)$ and an electrode adapted to the proximal left PV $(n=10)$. The cervical vagus nerve was stimulated with bipolar platinum electrodes $(20 \mathrm{~Hz})$. Epicardial activation was recorded in sinus rhythm, and effective refractory periods (ERPs), dispersion of refractoriness and conduction times from high-lateral right atrium (RA) to high-lateral left atrium (LA) and PVs assessed at baseline and during vagal stimulation. Burst pacing $(50 \mathrm{~Hz}, 10 \mathrm{~s})$, alone or combined with vagal stimulation, was applied to the right (RAA) and left atrial appendage (LAA) and PVs to induce AF. At baseline, ERPs were lower in PVs than in LA and LAA, but did not differ significantly from RA and RAA, and there was a significant delay in the conduction time from RA to PVs compared with the activation time from RA to LA $(P<0.01)$. During vagal stimulation, ERP decreased significantly at all sites, without significant differences in the dispersion of refractoriness, and the atrial conduction times changed from $39 \pm 19$ to $49 \pm 9 \mathrm{~ms}$ (RA to PVs; n.s.) and from $14 \pm 7$ to $28 \pm 12 \mathrm{~ms}$ (RA to LA; $P=0.01)$. Induction of AF was reproducible in $50 \%$ of cases with $50 \mathrm{~Hz}$ and in $82 \%$ with $50 \mathrm{~Hz}$ combined with vagal stimulation $(P<0.05)$. During vagal stimulation, AF cycle length decreased at all sites, and AF duration changed from $1.0 \pm 0.9$ to $14.0 \pm 10.0 \mathrm{~s}(P<0.01)$, with documentation of PV tachycardia in three cases. In $70 \%$ of the animals, AF ceased immediately after interruption of vagal stimulation. We conclude that in the intact rabbit heart, vagal activity prolongs interatrial conduction and shortens atrial and PV ERP, contributing to the vulnerability to the induction and maintenance of AF. This model may be useful in the assessment of the autonomic influence in the mechanisms underlying AF.
\end{abstract}

(Received 26 March 2010; accepted after revision 12 October 2010; first published online 15 October 2010)

Corresponding author M. Oliveira: Unit of Autonomic Nervous System, Institute of Physiology, Faculty of Medicine of Lisbon, Av Prof Egas Moniz, Lisbon 1640-028, Portugal. Email: m.martinsoliveira@gmail.com

Atrial fibrillation (AF) is the most common sustained cardiac arrhythmia in clinical practice, with impact on both life expectancy and quality of life (Camm, 1997; Benjamin et al. 1998; Van Den Berg et al. 2001). The pathophysiology of AF remains a considerable challenge, due to our limitations in understanding the precise mechanisms underlying the initiation and maintenance of this arrhythmia. Experimental work has provided an important contribution to improve comprehension of complex AF mechanisms. The relative contribution of the multiple wavelet hypothesis proposed by Moe et al. (1964) and the focal sources of electrical activity described 
by Haissaguerre et al. (1998) in explaining AF remain unknown. Nevertheless, phenomena that are able to influence electrophysiological properties of the atria can have a role in the pathogenesis of AF (Nattel, 2003).

The autonomic nervous system (ANS) is well recognized as an important modulator in mediating $\mathrm{AF}$ (Tai, 2001; Chen et al. 2006; Chen \& Tan, 2007). Variations of autonomic tone may be important in the genesis of paroxysmal AF. A number of electrophysiological properties related to atrial vulnerability to AF may change as a result of vagal or sympathetic activation. Vagal stimulation reduces the velocity of the conduction in the atrial tissue and shortens the action potential duration and atrial effective refractory periods (ERPs) non-uniformly, facilitating re-entrant arrhythmias (Nattel et al. 2000; Chen et al. 2006), and sympathetic stimulation can favour reduction of atrial refractoriness and induction of trigger activity (Nattel et al. 2000). However, the specific contribution of each division of the ANS to the genesis of AF remains unclear. Sharifov et al. (2004) reported that direct infusion of acetylcholine into the sinus node artery could induce AF in $100 \%$ of dogs, but infusion of isoprenaline and adrenaline induced AF only in $21 \%$ of the animals. Sympathetic outflow does not promote $\mathrm{AF}$ as much as vagal stimulation, probably because the effects of sympathetic stimulation are more spatially homogeneous in the atria than those resulting from vagal activity (Olshansky, 2005). It has been recognized that vagal activity might predispose patients to develop paroxysmal AF (Coumel et al. 1978), that vagal overactivity is a precipitating cause of several short daily attacks of an arrhythmia which are usually not completely nocturnal, and that vagal nerve stimulation can facilitate the induction of AF (Hirose et al. 2002). Characterization and quantification of ANS electrophysiological effects are complex and difficult to define. Several animal models have been developed to understand the origin and possible mechanisms underlying AF. Nevertheless, an important part of these experimental studies were carried out in isolated heart models. In the present study, we used the intact anaesthetized rabbit heart, with preserved autonomic innervation, in order to investigate the effects of vagal stimulation on atrial conduction velocity and refractoriness, and its relevance in the context of vulnerability for the induction and maintenance of AF.

\section{Methods}

\section{Ethical issues}

All the experimental procedures were performed in accordance with the Portuguese and European law on animal welfare and were also approved by the Ethics Committee of the Faculty of Medicine of Lisbon.

\section{Anaesthesia and surgical protocol}

Twentytwo New Zealand White rabbits of both sexes (weight $3.9-5.0 \mathrm{~kg}$ ) were anaesthetized with sodium pentobarbital $\left(60 \mathrm{mg} \mathrm{kg}^{-1}\right.$, I.V.). A tracheal cannula was inserted below the larynx, via a tracheostomy, to allow ventilation with $\mathrm{O}_{2}$-enriched room air through a positive-pressure ventilator (Harvard Apparatus, UK). Neuromuscular blockade was achieved with vecuronium $\left(0.05 \mathrm{mg} \mathrm{kg}^{-1} \mathrm{~h}^{-1}\right.$, I.v.), in order to assure strict control of respiratory parameters, such as rate and volume. Ventilation was adjusted to ensure an end-tidal $\mathrm{CO}_{2}$ of $5-7 \%$. Adequate levels of anaesthesia were maintained with a $20 \%$ solution of the anaesthetic and assessed by observing the presence of the withdrawal reflex after pinching a paw before the injection of vecuronium and from the observed changes in arterial blood pressure (BP) and heart rate after the same type of noxious stimulation following induction of neuromuscular blockade. The right femoral artery and vein were cannulated for BP monitoring (Lectromed, Letchworth, UK) and for intravenous drug and saline administration, respectively. Rectal temperature was monitored and maintained at $38 \pm 1^{\circ} \mathrm{C}$ throughout the experiment with a heating blanket (Harvard Apparatus). The urinary bladder was cannulated and drained.

A mid-line sternectomy was performed and the pericardium opened in order to expose the heart and prepare an epicardial approach. After thoracotomy, an end-expiratory pressure of $1-2 \mathrm{cmH}_{2} \mathrm{O}$ was maintained. The ECG was recorded with the use of bipolar percutaneous electrodes placed in the four limbs of the animal. Arterial blood pressure, ECG, heart rate, ventilatory rate and end-tidal $\mathrm{CO}_{2}$ were continuously monitored (PowerLab, ADInstruments, Chalgrove, UK). At the end of the experiment, the animal was killed with an overdose of sodium pentobarbital.

\section{Experimental protocol}

Electrical stimulation of the vagus nerve. The right cervical vagus nerve was identified and isolated via a midline neck incision and prepared for electrical stimulation with a bipolar silver electrode. As a physiological test, the vagus nerve was stimulated $(20 \mathrm{~Hz}, 0.2 \mathrm{~ms}, 150-200 \mu \mathrm{A})$ to evoke a sinus rate reduction of $50 \%$.

Electrophysiological protocol. Atrial electrograms were recorded in all animals from an array of four custommade unipolar electrodes $(125 \mu \mathrm{m} \mathrm{Ag-AgCl} \mathrm{Teflon-}$ isolated wire), inserted into a patch of nylon mesh at interelectrode distances of $4 \mathrm{~mm}$ (Fig. 1). This electrode array was placed epicardially and distributed along the atria, from the high-lateral right atrium (RA) to the highlateral left atrium (LA). In 10 animals, a fifth electrode (twin coaxial cable) was adapted to the left pulmonary 


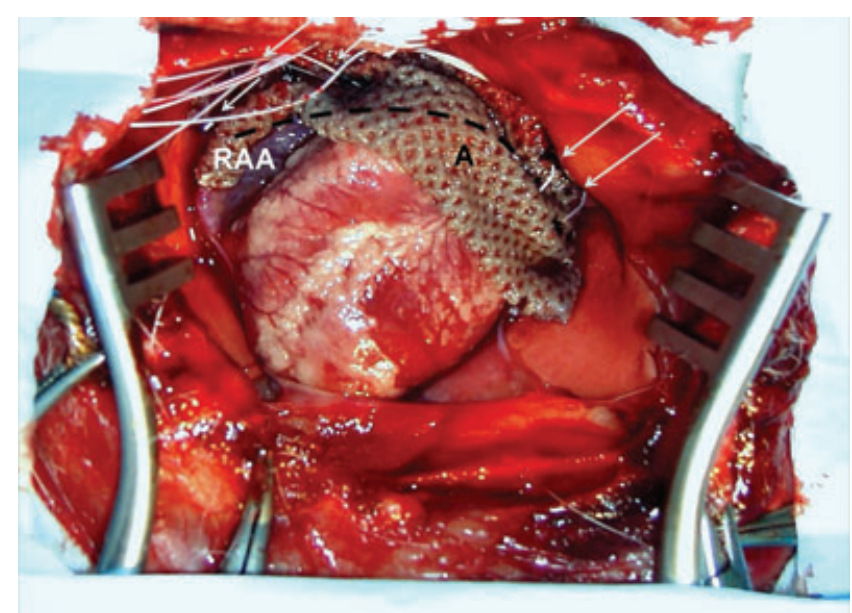

Figure 1. Open-chest preparation of the intact rabbit heart The nylon network (A) supports four unipolar electrodes (arrows) to record electrograms along the atria (from the high-lateral right atrium to the high-lateral left atrium. Abbreviation: RAA, right atrial appendage. veins (PVs), near the area of insertion in the LA, for recording of electrograms and pacing. Atrial epicardial activation was recorded during normal sinus rhythm, and the conduction time between RA and LA and between RA and PVs measured in baseline conditions and during vagal stimulation. The recorded signals were amplified, filtered at $0.5-300 \mathrm{~Hz}$ (Neurolog, Digitimer, Welwyn Garden City, UK) and digitized (PowerLab, ADInstruments; Fig. 2).

Bipolar electrical stimulation was performed with a Teflon-coated silver wire electrode $(0.1 \mathrm{~mm}$ diameter $)$, positioned with a micromanipulator (WPI, M330), and with a coaxial electrode adapted to the PVs for the assessment of ERPs and induction of AF. An eight-channel, programmable stimulator (Master8, AMPI, Israel) was used for pacing. As a measure of local refractoriness, ERPs were assessed in each animal at four different sites [RA, right atrial appendage (RAA), left atrial appendage (LAA) and LA]. Effective refractory periods of the PVs were also measured in 10 animals. In stable conditions, after all the surgical procedures and under
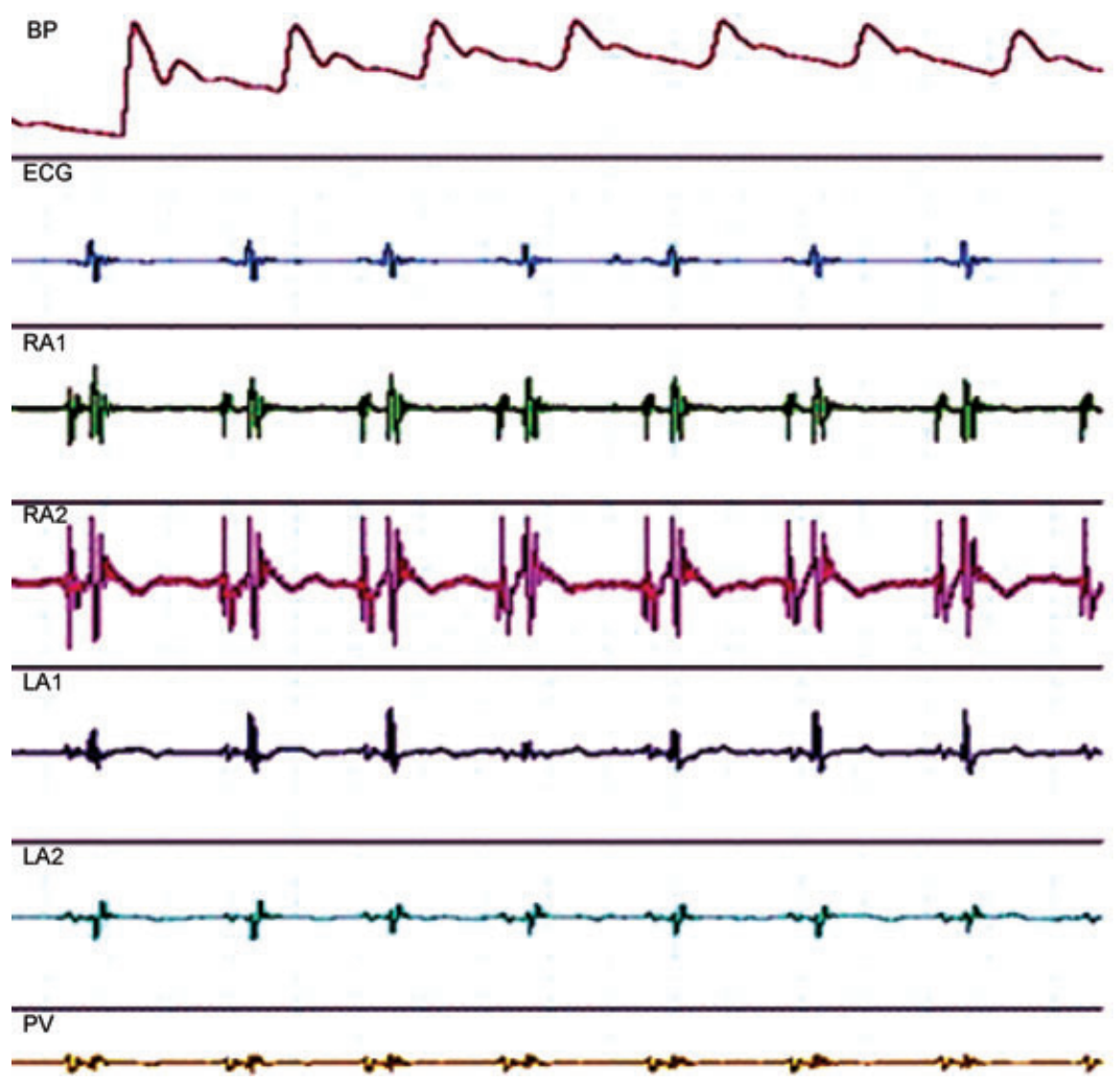

Figure 2. Continuous recording of blood pressure (BP), ECG, atrial and left pulmonary vein epicardial electrograms during sinus rhythm

Abbreviations: RA1, high-lateral right atrium; RA2, right atrial appendage; LA1, left atrial appendage; LA2, highlateral left atrium; and PV, pulmonary veins. 
neuromuscular blockade, heart rate ranged between 175 and 222 beats $\min ^{-1}\left(195 \pm 29\right.$ beats $\left.\min ^{-1}, n=22\right)$, the systolic BP between 60 and $90 \mathrm{mmHg}(82 \pm 15 \mathrm{mmHg}$, $n=22)$, and the $P$ wave between 38 and $60 \mathrm{~ms}(53 \pm 8 \mathrm{~ms}$, $n=22$ ). Vagal stimulation at the cervical level for $5 \mathrm{~s}$ elicited a pronounced sinus bradycardia, with occurrence of systemic hypotension $(<60 \mathrm{mmHg})$ in all animals.

Atrial and PV stimulation was performed with impulses of $2 \mathrm{~ms}$ duration at twice the diastolic threshold. A programmed electrical stimulation using a single premature stimulus was delivered, while pacing continuously at a basic drive cycle length of $300 \mathrm{~ms}$ (or 20 ms below the R-R interval in six rabbits with heart rate $>200$ beats $\mathrm{min}^{-1}$ ). A premature beat was introduced in late diastole, beginning at a coupling interval of $100 \mathrm{~ms}$ less than the basic cycle length. The coupling interval of the premature stimulation was decreased by $10 \mathrm{~ms}$ steps until the ERP was reached. The ERP was defined as the longest S1-S2 interval that failed to initiate a propagation response, and measured in baseline conditions and during vagal stimulation. Dispersion of atrial refractoriness was calculated as the difference between the longest and the shortest ERP at the stimulation sites. The AF cycle length was measured in all sites with online calipers at a paper speed of $100 \mathrm{~mm} \mathrm{~s}^{-1}$ by averaging five consecutive cycles before the termination of AF.

The concept of vulnerability to AF induction was evaluated with bursts $(50 \mathrm{~Hz}, 10 \mathrm{~s}$; supraliminal intensity), delivered with a bipolar electrode placed at the right and left atrial appendages, alone or combined with vagal stimulation. Atrial fibrillation was defined as a fast atrial rhythm characterized by a fluctuating beat-to-beat

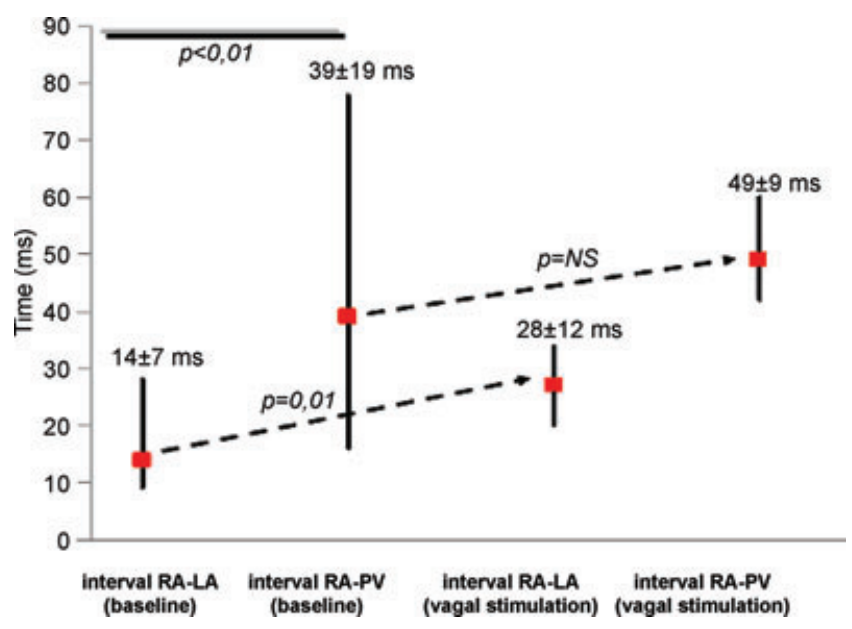

Figure 3. Atrial conduction times

Atrial conduction times between the high-lateral right atrium (RA) and the high-lateral left atrium (LA) and between the RA and left pulmonary veins (PV) near the area of insertion in the LA in baseline conditions and during right cervical vagal stimulation. ${ }^{*} p<0.01$ vs. interval RA-LA; ${ }^{*} p=0.01$ vs. baseline; $\S p=$ NS vs. baseline
Table 1. Effective refractory periods (ERPs) measured during baseline conditions and during vagal stimulation

\begin{tabular}{lcccc}
\hline & $\begin{array}{c}\text { Baseline } \\
\text { ERP }(\mathrm{ms})\end{array}$ & $\begin{array}{c}\text { ERP during vagal } \\
\text { stimulation (ms) }\end{array}$ & $\% \_\Delta$ & $P$ value \\
\hline $\begin{array}{l}\text { Right atrium } \\
\text { Right atrial }\end{array}$ & $86 \pm 17$ & $60 \pm 20$ & $-33 \pm 20 \%$ & $<0.01$ \\
$\begin{array}{l}\text { appendage } \\
\text { Left atrial }\end{array}$ & $105 \pm 24^{*}$ & $76 \pm 25$ & $-20 \pm 17 \%$ & $<0.05$ \\
$\begin{array}{l}\text { appendage } \\
\text { Left atrium }\end{array}$ & $102 \pm 27^{*}$ & $76 \pm 23$ & $-26 \pm 15 \%$ & $<0.01$ \\
$\begin{array}{l}\text { Pulmonary } \\
\text { veins }\end{array}$ & $65 \pm 40$ & $38 \pm 28$ & $-31 \pm 36 \%$ & $<0.01$ \\
\hline
\end{tabular}

Definition: \%_ $\Delta$, percentage of variation of the average ERPs. ${ }^{*} P<0.01$ compared with high-lateral RA, RAA and PVs in baseline conditions.

cycle length, polarity, configuration and amplitude of the recorded atrial electrograms and lasting more than five cycles (Hirose et al. 2002).

\section{Statistical analysis}

Results are shown as means \pm S.D. Categorical variables are expressed as frequencies and percentages. Normality of the distributions of the continuous variables was analysed with the Kolmogorov-Smirnov test. Student's paired $t$ test was used to assess all paired data in the same group. Comparisons between groups were made using Student's unpaired $t$ test or non-parametric analysis with the Mann-Whitney $U$ test, as appropriate. The $\chi^{2}$ test, with Yates correction, was used to evaluate the differences in categorical variables. All statistical tests were twosided, and a probability value $<0.05$ was required for statistical significance. Data were analysed using GraphPad Instat, version 3.05 (GraphPad Software, Inc., La Jolla, CA, USA).

\section{Results}

\section{Effects of vagal stimulation on electrophysiological parameters of the atria}

The conduction time from the RA to the LA in baseline conditions was $14 \pm 7 \mathrm{~ms}$ (10-30 ms), which increased to $28 \pm 10 \mathrm{~ms}(20-35 \mathrm{~ms})$ on stimulation $(20 \mathrm{~Hz}, 0.2 \mathrm{~ms}$, $150-200 \mu \mathrm{A})$ of the right vagus nerve $(n=22, P<0.05$; Fig. 3). In baseline conditions, during normal sinus rhythm, there was a significant delay in conduction between the RA and the PVs, slightly influenced by vagal stimulation (Fig. 3).

Table 1 shows the ERPs obtained in baseline conditions and during vagal activation. In baseline conditions, there was a significant increase of atrial refractoriness from the RA, RAA and PVs to the LA and LAA. Vagal stimulation produced a reproducible reduction in the atrial ERPs assessed in all sites. 
Table 2. Mean atrial fibrillation cycle lengths for atria and pulmonary veins measured in control conditions and following vagal stimulation

\begin{tabular}{lccccc}
\hline & $\begin{array}{c}\text { High-lateral } \\
\text { right atrium }\end{array}$ & $\begin{array}{c}\text { Right atrial } \\
\text { appendage }\end{array}$ & $\begin{array}{c}\text { High-lateral } \\
\text { left atrium }\end{array}$ & $\begin{array}{c}\text { Left atrial } \\
\text { appendage }\end{array}$ & $\begin{array}{c}\text { Proximal segment of } \\
\text { pulmonary veins }\end{array}$ \\
\hline Baseline AFCL (ms) & $154 \pm 35$ & $170 \pm 23$ & $173 \pm 16$ & $171 \pm 16$ & $152 \pm 25$ \\
AFCL during vagal stimulation (ms) & $83 \pm 34^{*}$ & $77 \pm 21^{* *}$ & $86 \pm 35^{* *}$ & $110 \pm 48 \S$ & $71 \pm 17^{* *}$ \\
\hline
\end{tabular}

Definition: AFCL, atrial fibrillation cycle length. ${ }^{*} P=0.002,{ }^{* *} P<0.0001, \S P<0.05$ for the comparison between control conditions and following vagal stimulation. Measurements obtained at different sites in the same conditions were not significantly different from each other.

The dispersion of ERPs based only on atrial measurements was significantly lower than the dispersion of ERPs calculated with atrial and PV refractoriness values $(33 \pm 14$ versus $64 \pm 25 \mathrm{~ms}, P<0.05)$. Compared with baseline conditions, vagal stimulation did not significantly affect the dispersion of refractoriness $(33 \pm 14$ versus $38 \pm 17 \mathrm{~ms}$ for atrial sites, n.s.; and $64 \pm 25$ versus $67 \pm 28 \mathrm{~ms}$ for all sites, n.s.).

\section{Effects of vagal stimulation on atrial fibrillation inducibility}

The inducibility and the duration of AF were significantly increased by vagal stimulation (Fig. 4). Atrial fibrillation was induced in $50 \%$ of the rabbits with rapid atrial bursts delivered to the RAA or PVs, but could not be induced by LAA high-rate pacing alone. During vagal activation, AF was consistently induced in 100, 60 and $20 \%$ of the rabbits by pacing the RAA, PVs and LAA, respectively (RAA versus LAA, $P<0.05$ ). The mean AF cycle length measured in baseline conditions and during vagal stimulation is represented in Table 2. The cycle lengths during AF induced by $50 \mathrm{~Hz}$ pacing were reduced significantly by vagal stimulation in all atrial sites and PVs. On three occasions, AF was initiated by a single extrastimulus combined with vagal stimulation during ERP measurements. Pulmoary vein tachycardia (cycle length $80-90 \mathrm{~ms}$ ) was induced by high-rate PV pacing combined with vagal stimulation in three animals (Fig. 5). The duration of the inducible AF changed from $1.0 \pm 0.8 \mathrm{~s}$ with $50 \mathrm{~Hz}$ rapid stimulation alone to $14.0 \pm 10.0 \mathrm{~s}$ in response to $50 \mathrm{~Hz}$ rapid stimulation combined with vagal stimulation $(P<0.01$; Fig. 4$)$. Atrial fibrillaion lasted $>10$ s in $45.5 \%$ of the rabbits (only during vagal stimulation) and terminated immediately after the cessation of vagal stimulation in seven of those 10 cases. These animals had similar interatrial and atrial to PV activation times. However, they showed the shortest ERPs in the RA, with higher dispersion of atrial refractoriness, at baseline and also during vagal stimulation (Table 3).

\section{Discussion}

The present experimental in vivo model allowed investigation of the effects of vagus nerve stimulation on cardiac electrophysiological properties and on the vulnerability to AF. We have characterized the influence of direct acute stimulation of the right cervical vagal trunk, the effect of which on heart rate is much larger than for identical left vagal stimulation (Loeb et al. 1981), on the electrical conduction and refractoriness properties of the atria and PVs. We have also confirmed a role of vagal activity in the vulnerability to the initiation of AF and its duration.

\section{Main findings}

This model shows a significant activation delay from the atria to the PVs during sinus rhythm. In fact, the mean conduction times from the RA to the PVs were about three times greater than the RA to LA interval measured in baseline conditions (Fig. 3). Also, a significant increase of baseline atrial ERPs was observed from the RA to the LA, suggesting heterogeneity of the electrophysiological properties of the atria. In addition, ERPs of the PV were significantly lower than those measured in

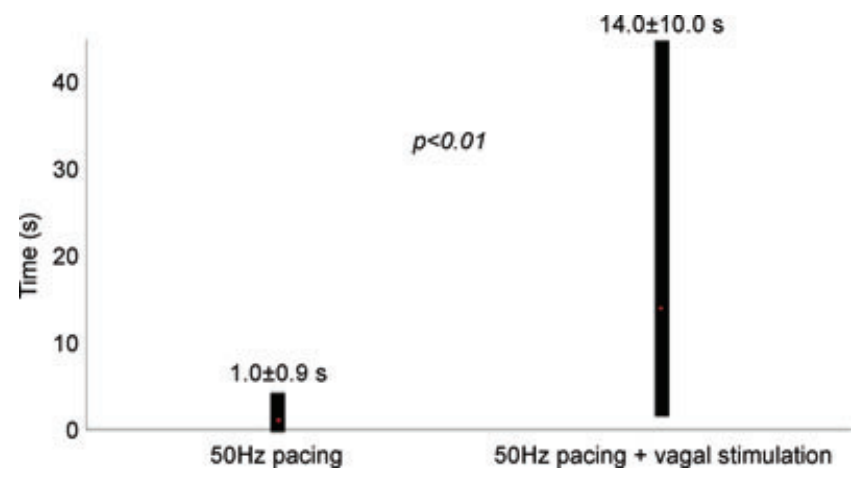

Figure 4. Duration of inducible atrial fibrillation episodes Duration of the inducible atrial fibrillation episodes with rapid atrial stimulation alone $(50 \mathrm{~Hz}$ pacing) and in response to rapid atrial stimulation combined with vagal activation $(50 \mathrm{~Hz}$ pacing + vagal stimulation). 
the LA and LAA. In this preparation, vagal activity was important for obtaining significant reproducible changes in atrial conduction times, atrial and PV ERPs, frequency of $\mathrm{AF}$ induction, mean $\mathrm{AF}$ cycle length and duration of the induced AF. Moreover, we demonstrated significant differences in atrial dispersion of refractoriness and RA ERPs in the group of animals with AF lasting $>10 \mathrm{~s}$.

\section{Animal models and mechanisms of atrial fibrillation}

Various animal models have been developed to explore the mechanisms underlying AF. The classic hypothesis of multiple circuit re-entry with variable spatial orientation, focal activity and single rotors with fibrillatory conduction, which remains the framework for our understanding of AF, was established based on experimental studies in animals. However, the development of models that allow electrophysiological studies and a contribution to the comprehension of the conditions associated with the generation of arrhythmias remains a major challenge. In dogs, microreentry, abnormal automaticity and triggered activity have been observed as possible mechanisms for the occurrence of atrial arrhythmias (Friedman et al. 1996; Hocini et al. 2002). Hayashi et al. (1998) used efferent cervical vagal stimulation to induce AF by bursts of atrial pacing in anaesthetized open-chested dogs and evaluated the efficacy of class Ic and class III antiarrhythmic drugs to terminate AF. Other investigators have used versions of this model to explore the ability of different antiarrhythmics to terminate induced AF (Goldberger \& Pavelec, 1986; David et al. 1990). Nevertheless, data regarding the use of experimental models with preserved autonomic innervation to assess the fundamental aspects of the electrophysiological properties of the atria and PVs underlying the vulnerability to development of $\mathrm{AF}$ are still limited. Abnormal atrial electrophysiology and higher vagal reflex activity may play an important role in the genesis of AF (Chen et al. 1999). In fact, it has been accepted that the ANS contributes as a modulator of the initiation, perpetuation, ventricular response rate and termination of $\mathrm{AF}$, but its precise role remains controversial (Olshansky, 2005; Vos et al. 2008). The

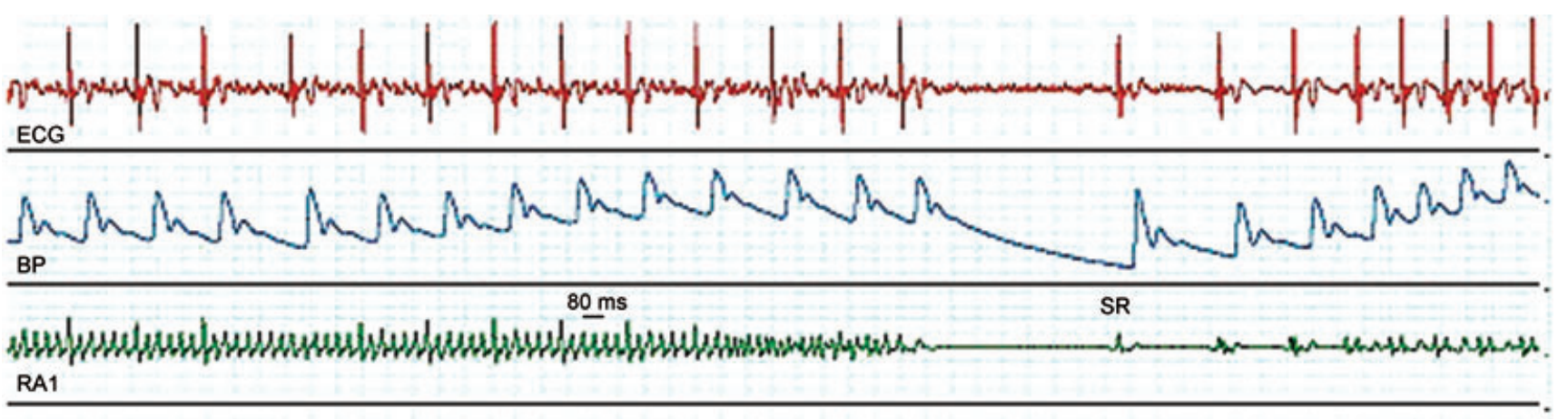

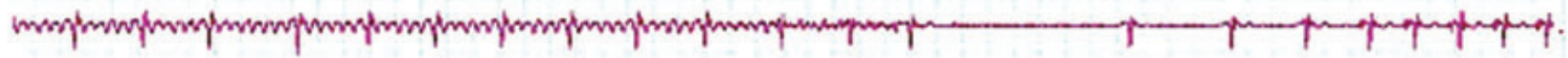
RA2

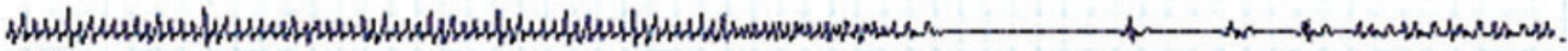
LA1

LA2

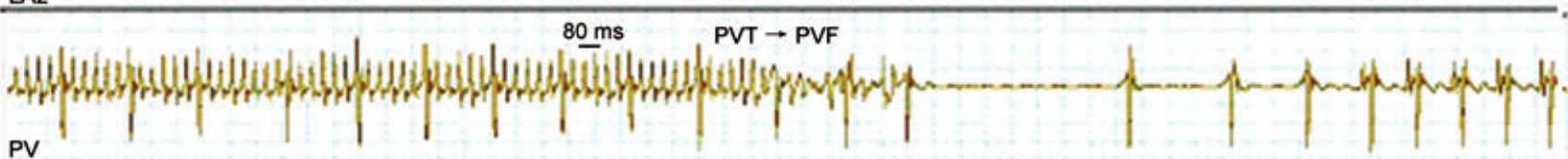

Figure 5. Example of a recording with pulmonary vein tachycardia (PVT) induced by stimulation of the pulmonary veins $(50 \mathrm{~Hz}, 10 \mathrm{~s})$ combined with vagal activation, showing the transition to pulmonary vein fibrillation (PVF) followed by termination of the arrhythmia

Abbreviations: BP, blood pressure; ECG, electrocardiogram; LA1, left atrial appendage; LA2, high-lateral left atrium; PV, pulmonary veins; PVF, pulmonary veins fibrillation; PVT, pulmonary veins tachycardia; RA1, high-lateral right atrium; RA2, right atrial appendage; and SR, sinus rhythm. 


\begin{tabular}{|c|c|c|c|}
\hline Parameter & Group AF $<10 \mathrm{~s}$ & Group $A F>10 \mathrm{~s}$ & $P$ value \\
\hline P wave duration (ms) & $35 \pm 9$ & $50 \pm 14$ & n.s. $(0.08)$ \\
\hline Baseline heart rate (beats $\min ^{-1}$ ) & $196 \pm 13$ & $208 \pm 28$ & n.s. \\
\hline Baseline systolic BP $(\mathrm{mmHg})$ & $78 \pm 11$ & $75 \pm 15$ & n.s. \\
\hline Weight (kg) & $4,4 \pm 0,3$ & $4,3 \pm 0,4$ & n.s. \\
\hline Atrial dispersion of ERP baseline (ms) & $22 \pm 15$ & $42 \pm 5$ & 0.03 \\
\hline Atrial + PVs dispersion of ERP baseline (ms) & $73 \pm 38$ & $60 \pm 26$ & n.s. \\
\hline Atrial dispersion of ERP vagal (ms) & $28 \pm 15$ & $48 \pm 11$ & 0.03 \\
\hline Atrial + PVs dispersion of ERP vagal (ms) & $72 \pm 40$ & $63 \pm 13$ & n.s. \\
\hline RA ERP baseline (ms) & $97 \pm 12$ & $70 \pm 10$ & $<0.05$ \\
\hline RA ERP vagal (ms) & $80 \pm 20$ & $40 \pm 10$ & $<0.05$ \\
\hline RAA ERP baseline (ms) & $67 \pm 15$ & $80 \pm 11$ & n.s. \\
\hline RAA ERP vagal (ms) & $53 \pm 15$ & $64 \pm 27$ & n.s. \\
\hline LAA ERP baseline (ms) & $102 \pm 21$ & $107 \pm 6$ & n.s. \\
\hline LAA ERP vagal & $75 \pm 24$ & $88 \pm 19$ & n.s. \\
\hline LA ERP baseline (ms) & $105 \pm 35$ & $100 \pm 27$ & n.s. \\
\hline LA ERP vagal (ms) & $80 \pm 56$ & $73 \pm 16$ & n.s. \\
\hline PVs ERP baseline (ms) & $75 \pm 51$ & $58 \pm 38$ & n.s. \\
\hline PVs ERP vagal (ms) & $41 \pm 33$ & $34 \pm 30$ & n.s. \\
\hline Interval RA-LA baseline (ms) & $10 \pm 7$ & $17 \pm 4$ & n.s. \\
\hline Interval RA-LA vagal (ms) & $26 \pm 9$ & $31 \pm 16$ & n.s. \\
\hline Interval RA-PVs baseline (ms) & $55 \pm 50$ & $40 \pm 20$ & n.s. \\
\hline Interval RA-PVs vagal (ms) & $58 \pm 20$ & $51 \pm 8$ & n.s. \\
\hline \multicolumn{4}{|c|}{$\begin{array}{l}\text { Definitions: AF, atrial fibrillation; } B P \text {, blood pressure; ERP, effective refractory period; } \\
\text { PVs, left pulmonary veins; and vagal, during cervical vagal nerve stimulation. Atrial + PV } \\
\text { dispersion includes ERP measurements from high-lateral right (RA) and left atrium (LA), } \\
\text { right (RAA) and left atrial appendage (LAA) and PVs. }\end{array}$} \\
\hline
\end{tabular}

importance of the ANS in AF is supported by animal experiments and recent clinical studies showing that vagal denervation enhances the efficacy of circumferential PV ablation in preventing AF recurrence (Chen \& Tan, 2007).

Decreased atrial ERP and its spatial dispersion heterogeneity have been accepted to promote AF reinitiation and to provide a substrate for the re-entry of multiple wavelets to enhance the ability of the disorder to sustain itself (Nattel et al. 2000). In previous studies, sympathetic stimulation was much less effective than vagal stimulation in promoting AF and heterogeneity in atrial refractoriness (Liu \& Nattel, 1997; Chen et al. 1999). Vagus nerve stimulation has been associated with atrial ERP shortening and facilitation of AF inducibility in dogs (Arora et al. 2008).

Our present data, using the intact rabbit heart model, support the importance of vagal activity in determining significant acute changes of atrial refractoriness and its contribution to the induction and cycle length of AF. The calculated dispersion of atrial refractoriness has not been significantly modified by vagal stimulation. This may explain the inability to sustain AF despite maintenance of vagal activation. In fact, reproducible induction of AF during vagal stimulation was obtained in all animals with a significant increase in the duration of the episodes, but the longest duration of AF was $45 \mathrm{~s}$, lasting $>10 \mathrm{~s}$ only in $45.5 \%$ of the rabbits. Interestingly, those animals with longer AF duration showed higher dispersion of atrial refractoriness and shortest RA ERPs, despite similar conduction activation times. This emphasizes the role of refractoriness properties as important markers of vulnerability to AF. We were able to obtain conditions that decrease atrial wavelength, the product of ERP and conduction velocity, by significantly decreasing the ERPs and the conduction velocity of the atrial tissue, which permits multiple wavelets and promotes AF.

\section{Effects of vagal stimulation on conduction times, refractoriness and vulnerability to atrial fibrillation}

Electrophysiological abnormalities behind the genesis of AF include conduction disturbances, shortening of the ERP and increase in atrial dispersion of refractoriness (Fuster et al. 2001). Vagal tone may influence the refractoriness and conduction velocity in the atrial tissue, contributing to the vulnerability to the occurrence of AF (Chen \& Tan, 2007). However, the assessment of autonomic fluctuations before the onset of paroxysmal AF, measured with heart rate variability, has shown conflicting results (Chen et al. 2006). 
In the present study, the electrophysiological characterization of the recognized effects of vagal stimulation in conduction and refractoriness properties of the atria and PVs was clearly shown. Oh et al. (2006), using a canine model, demonstrated that the effects of vagal stimulation on atrial ERPs and AF inducibility were significantly changed immediately after epicardial fat pad ablation, suggesting that it is possible to achieve temporary suppression of the vagal tone in the atria. Recently, in a study with dogs, sustained AF could be induced easily during vagal nerve stimulation in vivo (Lemola et al. 2008). Zhang et al. (2009) also used burst stimulation of the atria and right cervical vagal stimulation to induce AF in dogs. Their study suggested that strong vagal stimulation facilitates AF inducibility. In our rabbit model, we have shown that vagal activation was able to decrease the atrial and PV cycle length during AF and significantly increase the duration of the induced AF episodes (Table 2 and Fig.4). Vagal stimulation significantly increased the interatrial activation times, without changes in the conduction velocity between atrial and PV activation (Fig. 3). This suggests non-uniformity of vagal responses, with evidence of regional differences in conduction delay, and supports the concept that the presence of anisotropic conduction properties and slow conduction at the area of the PV-LA junction may contribute to promote reentry formation and thus play a role as a substrate for the maintenance of AF (Kumagai et al. 2004; Atienza \& Jalife, 2007). Also, the vagal effect on the degree of ERP reduction was slightly higher in the RA, LA and PVs.

Vagal activity seemed to influence the termination of AF directly, since in $70 \%$ of those animals with AF duration $>10 \mathrm{~s}$ the arrhythmia stopped immediately after cessation of vagal stimulation. Therefore, despite the inability to demonstrate a significant impact on the dispersion of the ERP, vagal activation decreased the conduction velocity in the atria and evoked reductions of the ERP, contributing to the induction, duration and termination of AF.

\section{Limitations of the study}

Although programmed electrical stimulation with a single premature stimulus has been widely used for the study of atrial refractoriness properties, we believe that further studies, using different electrophysiological techniques, may be needed to confirm these findings, because the present method may be of relatively low resolution. Also, the low tendency of the rabbit atria to fibrillate and maintain the arrhythmia owing to its small size might contribute to the inability to sustain AF in this preparation. Although brief episodes of AF in the rabbit reflect an evidence of arrhythmic vulnerability, the precise mechanisms underlying AF maintenance may be different in humans. Further experimental studies using more accurate methods may be needed to elucidate the impact of autonomic innervation on the dynamics of recurrent sustained AF episodes.

\section{Conclusions}

The acute stimulation of parasympathetic activity exerts a reduction of atrial conduction velocity and decreases ERPs in multiple atrial sites and in the PVs. This experimental preparation also displays the impact of vagal stimulation in enhancing the propensity for the inducibility of AF, demonstrating an important role in the vulnerability to the occurrence of $\mathrm{AF}$ and contributing to the duration of AF episodes, particularly in those animals with higher dispersion of refractoriness. Our data suggest that this experimental vagal stimulation model may provide a useful method for assessment of the influence of the ANS in the pathophysiology of AF.

\section{References}

Arora R, Ulphani JS, Villuendas R, Ng J, Harvey L, Thordson S, Inderyas F, Lu Y, Gordon D, Denes P, Greene R, Crawford S, Decker R, Morris A, Goldberger J \& Kadish AH (2008). Neural substrate for atrial fibrillation: implications for targeted parasympathetic blockade in the posterior left atrium. Am J Physiol Heart Circ Physiol 294, H134-H144.

Atienza F \& Jalife J (2007). Reentry and atrial fibrillation. Heart Rhythm 4, S13-S16.

Benjamin EJ, Wolf PA, D'Agostino RB, Silbershatz H, Kannel WB \& Levy D (1998). Impact of atrial fibrillation on the risk of death. The Framingham Heart Study. Circulation 98, 946-952.

Camm AJ (1997). Preface. In Nonpharmacological Treatment of Atrial Fibrillation, ed. Murgatroyd FD \& Camm AJ, pp. 536. Futura, Armonk, New York.

Chen J, Wasmund SL \& Hamdan MH (2006). Back to the future: the role of the autonomic nervous system in atrial fibrillation. Pacing Clin Electrophysiol 29, 413-421.

Chen P \& Tan AY (2007). Autonomic nerve activity and atrial fibrillation. Heart Rhythm 4, S61-S64.

Chen YJ, Tai CT, Chiou CW, Wen ZC, Chan P, Lee SH \& Chen SA (1999). Inducibility of atrial fibrillation during atrioventricular pacing with varying intervals: role of atrial electrophysiology and the autonomic nervous system. $J$ Cardiovasc Electrophysiol 10, 1578-1585.

Coumel P, Attuel P, Lavallee J, Flammang D, Leclercq JF \& Slama R (1978). The atrial arrhythmia syndrome of vagal origin. Arch Mal Coeur Vaiss 71, 645-656.

David D, Lang RM, Neumann A, Borow KM, Akselrod S \& Mor-Avi V (1990). Parasympathetically modulated antiarrhythmic action of lidocaine in atrial fibrillation. Am Heart J 119, 1061-1068. 
Friedman HS, Sinha B, Tun A, Pasha R, Sharafkhaneh A \& Bharadwaj A (1996). Zones of atrial vulnerability. Relationships to basic cycle length. Circulation 94, 1456-1464.

Fuster V, Rydén LE, Cannom DS, Crijns HJ, et al (2006). ACC/AHA/ESC 2006 Guidelines for the Management of Patients with Atrial Fibrillation. Circulation 114, e257-e354.

Goldberger A \& Pavelec R (1986). Vagally-mediated atrial fibrillation in dogs: conversion with bretilium tosylate. Int $\mathrm{J}$ Cardiol 13, 47-55.

Haissaguerre M, Jais P \& Shah DC (1998). Spontaneous initiation of atrial fibrillation by ectopic beats originating in the pulmonary veins. N Engl J Med 339, 659-666.

Hayashi H, Fujiki A, Tani M, Usui M \& Inoue H (1998). Different effects of class Ic and III antiarrhythmic drugs on vagotonic atrial fibrillation in the canine heart. J Cardiovasc Pharmacol 31, 101-107.

Hirose M, Leatmanoratn Z, Laurita R \& Carlson D (2002). Partial vagal denervation increases vulnerability to vagally induced atrial fibrillation. J Cardiovasc Electrophysiol 13, 1272-1279.

Hocini M, Ho SY, Kawara T, Linnenbank AC, Potse M, Shah D, Jaïs P, Janse MJ, Haïssaguerre M \& De Bakker JM (2002). Electrical conduction in canine pulmonary veins. Electrical and anatomic correlation. Circulation 105, 2442-2448.

Kumagai K, Ogawa M, Noguchi H, Yasuda T, Nakashima H \& Saku K (2004). Electrophysiologic properties of pulmonary veins assessed using a multielectrode basket catheter. $\mathrm{J} \mathrm{Am}$ Coll Cardiol 43, 2281-2289.

Lemola K, Chartier D, Yeh YH, Dubuc M, Cartier R, Armour A, Ting M, Sakabe M, Shiroshita-Takeshita A, Comtois P \& Nattel S (2008). Pulmonary vein region ablation in experimental vagal atrial fibrillation: role of pulmonary veins versus autonomic ganglia. Circulation 117, 470-477.

Liu L \& Nattel S (1997). Differing sympathetic and vagal effects on atrial fibrillation in dogs: role of refractoriness heterogeneity. Am J Physiol Heart Circ Physiol 42, H805-H816.
Loeb J, Dalton M \& Moran JM (1981). Sensitivity differences of SA and AV node to vagal stimulation: attenuation of vagal effects at SA node. Am J Physiol Heart Circ Physiol 241, H684-H690.

Moe GK, Rheinboldt WC \& Abilddskov JA (1964). A computer model of atrial fibrillation. Am Heart J 67, 200-220.

Nattel S (2003). Atrial electrophysiology and mechanisms of atrial fibrillation. J Cardiovasc Pharmacol Ther 8, S5-S11.

Nattel S, Li D \& Yue L (2000). Basic mechanisms of atrial fibrillation-very new insights into very old ideas. Annu Rev Physiol 62, 51-77.

Oh S, Zhang Y, Bibevski S, Marrouche NF, Natale A \& Mazgalev TN (2006). Vagal denervation and atrial fibrillation inducibility: epicardial fat pad ablation does not have long-term effects. Heart Rhythm 3, 701-708.

Olshansky B (2005). Interrelationships between the autonomic nervous system and atrial fibrillation. Prog Cardiovasc Dis 48, 57-78.

Sharifov OF, Fedorov VV, Beloshapko GG, Glukhov AV, Yushmanova V \& Rosenshtraukh L (2004). Roles of adrenergic and cholinergic stimulation in spontaneous atrial fibrillation in dogs. J Am Coll Cardiol 43, 483-490.

Tai CT (2001). Role of autonomic influences in the initiation and perpetuation of focal atrial fibrillation. J Cardiovasc Electrophysiol 12, 292-293.

Van Den Berg MP, Hassink RJ, Tuinenburg AE, van Sonderen EF, Lefrandt JD, de Kam PJ, van Gelder IC, Smit AJ, Sanderman R \& Crijns HJ (2001). Quality of life in patients with paroxysmal atrial fibrillation and its predictors: importance of the autonomic nervous system. Eur Heart J 22, 247-253.

Vos C, Nieuwlaat R, Crijns HJ, Camm J, LeHeuzey JY, Kirchhof C, Capucci A, Breithardt G, Vardas PE, Pisters R \& Tieleman RG (2008). Autonomic trigger patterns and anti-arrhythmic treatment of paroxysmal atrial fibrillation: data from the Euro Heart Survey. Eur Heart J 29, 632-639.

Zhang Y, Ilsar I, Sabbah H, David T \& Mazgalev T (2009). Relationship between right cervical vagus nerve stimulation and atrial fibrillation inducibility: therapeutic intensities do not increase arrhythmogenesis. Heart Rhythm 6, 244-250. 